Research Paper

\title{
PD-L1 Expression in Chinese Patients with Advanced Non-Small Cell Lung Cancer (NSCLC): A Multi-Center Retrospective Observational Study
}

Xin Yang ${ }^{1^{*}}$, Lili Jiang $2^{*}$, Yan Jin ${ }^{3^{*}}$, Peng $\mathrm{Li}^{4^{*}}$, Yingyong Hou ${ }^{5}$, Jingping Yun ${ }^{4}$, Chunyan $\mathrm{Wu}^{6}$, Wenyong Sun ${ }^{7}$, Xiangshan Fan ${ }^{8}$, Dong Kuang 9 , Weiya Wang ${ }^{2}$, Jinsong Ni ${ }^{10}$, Anhua Mao'11, Wenmin Tang ${ }^{11}$, Zhenhua Liu'11, Jiali Wang11, Suijun Xiao'11, Yuan $\mathrm{Li}^{1{ }^{\boxplus}}$, Dongmei Lin ${ }^{1 凶}$

1. Key Laboratory of Carcinogenesis and Translational Research (Ministry of Education), Department of Pathology, Peking University Cancer Hospital \& Institute, Beijing, China

2. Department of Pathology, West China Hospital, Sichuan University, Chengdu, Sichuan, China

3. Department of Pathology, Fudan University Shanghai Cancer Center, Shanghai, China

4. Department of Pathology, Sun Yat-sen University Cancer Center, Guangzhou, China

5. Zhongshan Hospital of Fudan University, Shanghai, China

6. Department of Pathology, Shanghai Pulmonary Hospital, Tongji University School of Medicine, Shanghai, China

7. Zhejiang Cancer Hospital, Hangzhou, Zhejiang, China

8. Nanjing Drum Tower Hospital, Nanjing, Jiangsu, China

9. Department of Pathology, Tongji Hospital, Tongji Medical College, Huazhong University of Science and Technology, Wuhan, Hubei, China

10. The first hospital of Jilin University - The Eastern Division, Changchun, Jilin, China

11. Medical Affairs Department, MSD China, Shanghai, China

* Authors contributed equally

$\square$ Corresponding authors: Prof. Dongmei Lin, Key Laboratory of Carcinogenesis and Translational Research (Ministry of Education), Department of Pathology, Peking University Cancer Hospital \& Institute, Beijing, China; lindm3@bjmu.edu.cn Prof. Yuan Li, Fudan University Cancer Hospital. 270 Dongan Road, Xuhui District, Shanghai, China; lumoxuan2009@163.com

(c) The author(s). This is an open access article distributed under the terms of the Creative Commons Attribution License (https://creativecommons.org/licenses/by/4.0/). See http://ivyspring.com/terms for full terms and conditions.

Received: 2021.05.21; Accepted: 2021.10.03; Published: 2021.10.28

\begin{abstract}
Objective: This study aimed to investigate the prevalence of tumor programmed death-ligand 1 (PD-LI) expression in Chinese patients with advanced Non-Small Cell Lung Cancer (NSCLC).

Methods: Tumor tissues with histologically confirmed stage IIIB/IV NSCLC were retrospectively obtained from 10 centers in China. PD-L1 expression was determined using the PD-LI IHC 22C3 pharmDx kit (Agilent, Santa Clara, CA, USA) and the samples were repetitively assayed with the PD-L1 IHC 22C3 Ab concentrate (Agilent, Santa Clara, CA, USA).

Results: Out of 901 patients who met the inclusion criteria, 879 (97.6\%) had evaluable PD-L1 data. The number of patients with a PD-L1 tumor proportion score (TPS) $<1 \%, 1-49 \%$, and $\geq 50 \%$ (corresponding to PD-L1 non-expression, low expression, and high expression) was 424 (48.2\%), 266 (30.3\%), and 189 (21.5\%), respectively. PD-L1 expression was more likely to be found in patients younger than 75 years, men, current or former smokers, those with good performance status (PS) scores, and those with a wild-type epidermal growth factor receptor (EGFR). PD-LI TPS $\geq 50 \%$ and $\geq 1 \%$ were respectively $28.0 \%$ and $50.2 \%$ among patients negative for both EGFR mutation and anaplastic lymphoma kinase (ALK) rearrangement. PD-Ll expression determined using the $22 \mathrm{C} 3$ antibody concentrate and pharmDx kit had comparable results.
\end{abstract}

Conclusions: The prevalence of PD-L1 expression in advanced NSCLC was consistent with that reported in the global EXPRESS study. Age, gender, smoking history, PS scores, and EGFR/ALK mutation status affected PD-L1 expression. The $22 \mathrm{C} 3$ antibody concentrate appears to be an alternative reagent for the PD-LI assay.

Key words: non-small cell lung cancer; programmed death-ligand 1; immunohistochemistry; 22C3 antibody; driver mutations 


\section{Introduction}

Lung cancer is the leading cause of cancer deaths globally $[1,2]$. However, the progress in precision therapy has contributed to a decline in related death rates. The annual mortality was reported to have declined by $5.9 \sim 6.3 \%$ in men and women with non-small cell lung cancer (NSCLC) since 2013 when biomarker-assisted targeted therapy was approved as a first-line regimen $[1,3]$. It is estimated that recent advances in immunotherapy will further boost the survival rates for lung cancer, especially for those without driver mutations [1]. Indeed, immune checkpoint inhibitor (ICI)-based immunotherapy has become an important treatment modality for advanced NSCLC ever since it was approved by the Food and Drug Administration (FDA) in 2015. Single-agent immunotherapy or combination immunotherapy has dramatically improved long-term survival for advanced NSCLC [4-8].

Biomarkers for immunotherapy are helpful in decision making and identifying candidates who are more likely to respond to ICIs such as anti-programmed-cell death-1 (PD-1) or its ligand (PD-L1) monoclonal antibodies. PD-L1 expression is a rational biomarker for predicting the response to immunotherapy and the positive correlation between tumor PD-L1 expression and treatment response has been described in numerous clinical trials [9]. The international KEYNOTE-001 study is the largest trial to validate the predictive value of tumor PD-L1 expression analyzed with an immunohistochemical (IHC) assay in lung cancer [10]. The companion diagnostic PD-L1 IHC 22C3 pharmDx ${ }^{\mathrm{TM}}$ was subsequently approved to select NSCLC patients eligible for treatment with the PD-1 inhibitor pembrolizumab [11]. For a panoramic understanding of the treatment candidates, the global EXPRESS study explored PD-L1 expression levels in NSCLC patients [12]. Several other studies revealed that the prevalence of PD-L1 expression varied depending on different diagnostic patterns and populations. A recent meta-analysis including 50 studies concluded that PD-L1 expression was associated with several clinicopathological features and may serve as a poor prognostic biomarker for patients with lung cancer [13]. However, limited data on PD-L1 expression are available for the Chinese NSCLC population, especially for the advanced stage.

The current study was conducted to obtain a profile of PD-L1 expression determined using 22C3 pharmDx ${ }^{\mathrm{TM}}$ in Chinese patients with advanced NSCLC. In addition, the performance of validated laboratory-developed tests (LDTs) using the 22C3 antibody concentrate with different platforms was evaluated in the study.

\section{Materials and methods}

\section{Study design and objectives}

This was a national, multicenter, retrospective, and observational study conducted in 10 centers in China aiming to evaluate the prevalence of PD-L1 protein on tumor tissue samples of advanced NSCLC.

The primary objective was to determine the prevalence of PD-L1 expression in Chinese patients with stage IIIB/IV NSCLC using the PD-L1 IHC 22C3 pharmDx ${ }^{\mathrm{TM}}$ kit. The secondary objectives were 1) to uncover the relationship between PD-L1 expression and baseline demographic, clinicopathological, and genetic (epidermal growth factor receptor (EGFR) / anaplastic lymphoma kinase (ALK)) characteristics; 2) to assess the feasibility of PD-L1 expression measurement using the $22 \mathrm{C} 3$ antibody concentrate.

\section{Patient eligibility}

The inclusion criteria were as follows: histologically confirmed primary stage IIIB/IV NSCLC; $\geq 18$ years at diagnosis; treatment-naive tumor tissues available for PD-L1 measurement; and formalin-fixed paraffin-embedded (FFPE) tumor tissue block no more than 3 years old. All participants signed informed consent forms or had conditions that were accepted by the Institutional Review Board (IRB)/Ethical Review Committee (ERC) for waiving ICF.

\section{PD-LI assessment}

\section{Sample}

A total of five 4-5 $\mu \mathrm{m}$ FFPE sections from resected tissue, excisional biopsies, or core needle biopsies were required for each patient. Minimally, one matched hematoxylin and eosin (H\&E) stained slide and two unstained slides for PD-L1 testing and the negative control were required. Samples were selected consecutively when possible.

\section{Measurement and platform}

PD-L1 expression was determined using the PD-L1 IHC 22C3 pharmDx kit (Agilent, Santa Clara, CA, USA) on the Dako ASL48 platform. A total of 5x 4-5 $\mu \mathrm{m}$ freshly cut (within 1 month of assay, preferably within 1 week) FFPE sections from excisional biopsies or resected tissue or core needle biopsies are required for each patient. Two unstained slides (one for PD-L1 testing and one as a negative control) and one matched H\&E stained slide are the minimum requirements; four unstained slides and one matched H\&E stained slide are preferred to allow 
for rapid retest in the event that this is needed. PD-L1 IHC $22 \mathrm{C} 3$ pharmDx ${ }^{\mathrm{TM}}$ is a qualitative IHC assay using monoclonal mouse anti-PD-L1, clone 22C3. It is intended for use with the EnVision FLEX visualization system on the Dako ASL48 platform. PD-L1 protein expression is determined using a tumor proportion score (TPS), which is the percentage of viable tumor cells showing partial or complete membrane staining.

PD-L1 IHC 22C3 Ab concentrate (Agilent, Santa Clara, CA, USA) was additionally applied to some of the samples for testing on the Dako ASL48 platform or the Ventana BenchMark platform. More specifically, specimens were sectioned at a thickness of $3 \mu \mathrm{m}$ and stained on positively charged glass slides stored at $4^{\circ} \mathrm{C}$ within 3 days of sectioning. Deparaffinization, rehydration, and antigen retrieval were performed on PT Link (Dako PT100) using the EnVision ${ }^{\text {TM }}$ FLEX Target Retrieval Solution at $\mathrm{pH} 6.0$ for 53 minutes at room temperature. Following FLEX peroxidase block for 5 minutes, specimens were incubated with primary mouse anti-PD-L1 monoclonal antibody (ref. M365329; Dako, Inc.) using a concentration of 1:50 for 60 minutes at room temperature. Specimens were then incubated with the EnVision ${ }^{\mathrm{TM}}$ FLEX+ Mouse LINKER for 30 minutes at room temperature, followed by incubation with the EnVision ${ }^{\mathrm{TM}}$ FLEX HRP visualization reagent for 30 minutes at room temperature. Enzymatic conversion of the subsequently added 3,3'-diaminobenzidine tetrahydrochloride (DAB) chromogen for 10 minutes at room temperature followed by $\mathrm{DAB}$ enhancer for 5 minutes at room temperature resulted in the precipitation of a visible reaction product at the site of the antigen. The specimens were then counterstained with hematoxylin and coverslipped. All described steps were followed by buffer washes for 5 minutes (EnVisionTM FLEX Wash Buffer 20x). Each IHC run contained a positive control (on-slide tonsil tissue) and a negative antibody control (buffer, no primary antibody).

\section{Quality control}

Scoring was performed by certified pathologists at each site who attended organized pathology training programs on the detection of PD-L1 IHC before the study. The pathologists were blinded to the method (22C3 pharmaDx Kit or 22C3 antibody concentrate) used on individual slides and the order of the samples was determined by a technician. The samples were categorized as TPS $\geq 50 \%$ (high PD-L1 expression), $1-49 \%$ (low PD-L1 expression), $\geq 1 \%$ (PD-L1 expression), or < 1\% (PD-L1 non-expression).

\section{Variables}

Demographics, clinicopathological, and biomarker data were collected and recorded in a central database for analysis. Key variables included age at diagnosis, gender, Eastern Cooperative Oncology Group Performance Status (ECOG PS), smoking status, center, tumor stage, histology, site and type of tumor tissue samples, EGFR mutation status, and ALK translocation status. Information on the EGFR or ALK genetic status was retrospectively extracted from each center's electronic medical records system or by chart review. EGFR mutation was commonly measured using amplification refractory mutation system-polymerase chain reaction (ARMS-PCR), digital PCR, and next-generation sequencing (NGS). ALK rearrangement was screened with Ventana IHC, fluorescent in situ hybridization (FISH), reverse transcriptase (RT)-PCR, and NGS.

\section{Statistical analyses}

Continuous data reported as the mean \pm standard deviation or median (interquartile range) were compared with the $t$-test for comparisons between the two subgroups or one-way analysis for comparisons among the three subgroups. Categorical data expressed as a number with percentage (\%) were compared with Pearson's Chi-Square test or Fisher's exact test if $\geq 25 \%$ of cells had expected counts $<5$. A p-value $<0.05$ was regarded as statistically significant.

\section{Results}

\section{Patient screening and baseline characteristics}

The study started on January 1, 2019, with the first center initiation on August 15, 2019. Analysis of the last sample took place on June 29, 2020. A total of 1023 patients were initially screened. Out of 901 tumor samples that met the inclusion criteria, 879 samples $(97.6 \%)$ had evaluable PD-L1 expression results and were included in the final analysis (Figure 1).

The median age of the overall population at diagnosis was 63.0 years (ranged: 30-90 years). The majority of the population were diagnosed at an age $<$ 75 years $(90.1 \%)$, were men $(63.9 \%)$, had never smoked (50.9\%), and had an ECOG PS score of 0-1 $(70.2 \%)$. The tumor samples were mostly at stage IV (71.0\%) and adenocarcinoma (71.7\%). The percentage of samples from primary tumor tissues was $66.4 \%$ and $49.6 \%$ were from the core needle biopsy. Samples with ALK rearrangement accounted for $3.6 \%$ and those with EGFR mutation accounted for $23.9 \%$ in the entire population (Table 1). Two hundred and forty-eight samples were repetitively tested using the 22C3 antibody concentrate. 


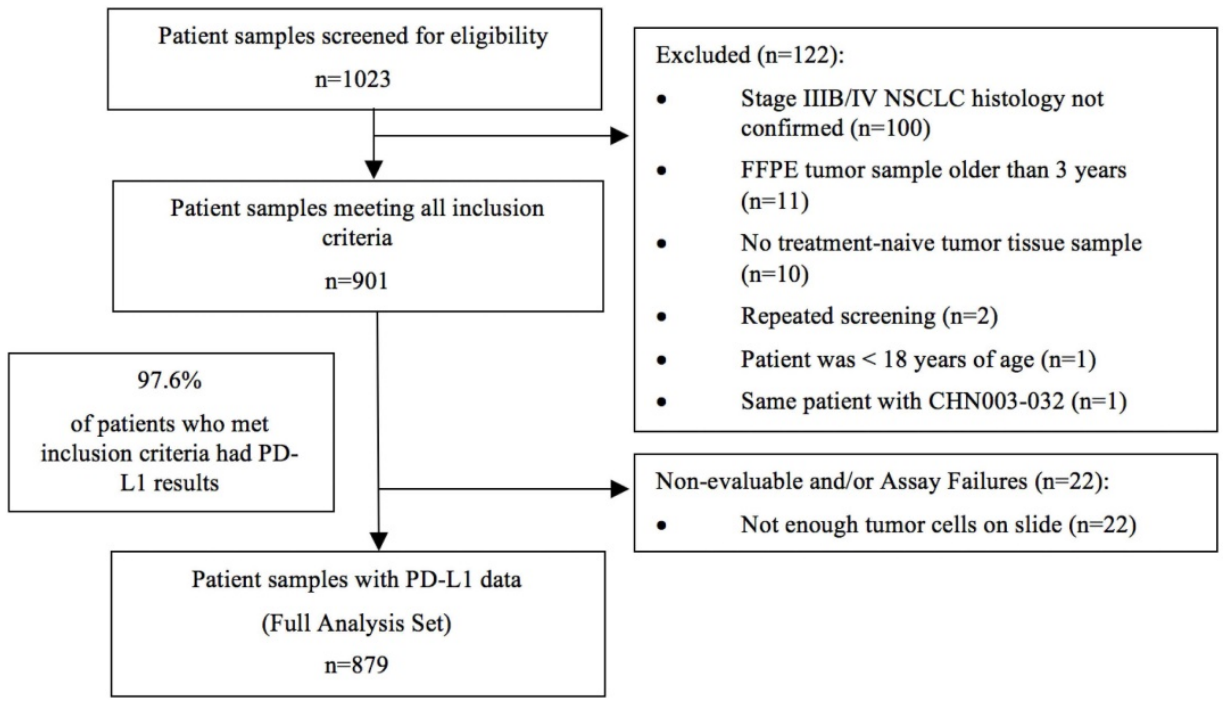

Figure 1. Study flow chart

Table 1. Demographic and clinicopathological characteristics of the study population $(\mathrm{N}=879)$

\begin{tabular}{|c|c|}
\hline Category & $\mathrm{N}(\%)$ \\
\hline \multicolumn{2}{|l|}{ Age at diagnosis } \\
\hline Median & 63.0 \\
\hline Min, Max & 30,90 \\
\hline \multicolumn{2}{|l|}{ Age at diagnosis } \\
\hline$<75$ & $792(90.1)$ \\
\hline$\geq 75$ & $87(9.9)$ \\
\hline \multicolumn{2}{|l|}{ Age at diagnosis } \\
\hline$<$ Median & $402(45.7)$ \\
\hline$\geq$ Median & $477(54.3)$ \\
\hline \multicolumn{2}{|l|}{ Gender } \\
\hline Male & $562(63.9)$ \\
\hline Female & $317(36.1)$ \\
\hline \multicolumn{2}{|l|}{ Race } \\
\hline Chinese & $878(99.9)$ \\
\hline Other & $1(0.1)$ \\
\hline \multicolumn{2}{|l|}{ Smoking status } \\
\hline Never & $447(55.8)$ \\
\hline Former & $209(26.1)$ \\
\hline Current & $145(18.1)$ \\
\hline \multicolumn{2}{|l|}{ ECOG Performance Status } \\
\hline $0-1$ & $617(92.6)$ \\
\hline$\geq 2$ & $49(7.4)$ \\
\hline \multicolumn{2}{|l|}{ AJCC Stage } \\
\hline IIIB & $225(25.6)$ \\
\hline IV & $624(71.0)$ \\
\hline Others & $30(3.4)$ \\
\hline \multicolumn{2}{|l|}{ Histology subtype } \\
\hline Squamous cell carcinoma & $207(23.5)$ \\
\hline Adenocarcinoma & $630(71.7)$ \\
\hline Others & $42(4.8)$ \\
\hline \multicolumn{2}{|l|}{ Site } \\
\hline Primary tumor & $584(66.4)$ \\
\hline Metastatic lesion & $295(33.6)$ \\
\hline \multicolumn{2}{|l|}{ Sampling location of metastatic lesion } \\
\hline Lymph node & $134(15.2)$ \\
\hline Brain & $58(6.6)$ \\
\hline Liver & $9(1.0)$ \\
\hline Others & $94(10.7)$ \\
\hline \multicolumn{2}{|l|}{ Tissue type } \\
\hline Surgical resection & $314(35.7)$ \\
\hline $\begin{array}{l}\text { Biopsy } \\
\text { (Core needle biopsy + Bronchial Biopsy + Pleura Biopsy + } \\
\text { EBUS + TBNA) }\end{array}$ & $511(58.1)$ \\
\hline Others & $54(6.1)$ \\
\hline ALK status & \\
\hline
\end{tabular}

\begin{tabular}{lc}
\hline Rearrangement & $32(6.0)$ \\
Wild type & $500(94.0)$ \\
EGFR mutation status & \\
Mutant & $210(43.1)$ \\
Wild type & $277(56.9)$ \\
Joint ALK/EGFR Status & \\
ALK+ or EGFR+ & $190(45.8)$ \\
ALK-/EGFR- & $225(54.2)$ \\
\hline ECOG: Eastern Cooperative Oncology Group; AJCC: American Joint Committee on \\
Cancer; EGFR: epidermal growth factor receptor; ALK: anaplastic lymphoma \\
kinase; EBUS-TBNA: endobronchial ultrasound-guided transbronchial fine needle \\
aspiration
\end{tabular}

\section{Prevalence of PD-LI expression}

The number of samples that had PD-L1 non-expression, low expression, and high expression, defined as TPS < 1\%, $1-49 \%$, and $\geq 50 \%$, was 424 (48.2\%), 266 (30.3\%), and $189(21.5 \%)$, respectively.

The prevalence of high PD-L1 expression (TPS $\geq$ $50 \%)$ was higher in patients aged $<75$ years $(22.7 \%)$ compared with the prevalence in older patients $(10.3 \%)$. Similarly, male patients were more likely to have high PD-L1 expression (25.6\%) than female patients $(14.2 \%)$. Current or former smokers tended to have a higher likelihood of exhibiting PD-L1 expression (TPS $\geq 1 \%$ : 58.5\%) than never-smokers $(47.0 \%)$. In addition, PD-L1 expression levels differed in patients with good and poor PS scores $(p=0.0044)$. A marginally significant difference in PD-L1 expression was observed in samples with different histology results, with squamous cell carcinoma presenting a numerically higher proportion of PD-L1 expression than the non-squamous type (TPS $\geq 1 \%$ : $58.9 \%$ vs. $49.6 \%$ ). The PD-L1 expression did not differ significantly in terms of TNM stage, primary or metastatic tissue, surgical or biopsy tissue, and different sampling locations (Table 2). 
Table 2. Prevalence of PD-L1 TPS by baseline demographic and clinicopathologic characteristics

\begin{tabular}{|c|c|c|c|c|}
\hline Category, N (\%) & TPS $<1 \%$ & TPS $1-49 \%$ & TPS $\geq 50 \%$ & $\mathrm{P}$-value \\
\hline Total & $424(48.2)$ & $266(30.3)$ & $189(21.5)$ & \\
\hline Age at diagnosis & & & & 0.0149 \\
\hline$<75$ & 379 (47.9) & $233(29.4)$ & $180(22.7)$ & \\
\hline$\geq 75$ & $45(51.7)$ & $33(37.9)$ & $9(10.3)$ & \\
\hline Gender & & & & 0.0002 \\
\hline Male & $250(44.5)$ & $168(29.9)$ & $144(25.6)$ & \\
\hline Female & $174(54.9)$ & $98(30.9)$ & 45 (14.2) & \\
\hline Smoking status & & & & $<0.0001$ \\
\hline Never & $237(53.0)$ & $140(31.3)$ & $70(15.7)$ & \\
\hline Current/Former & $147(41.5)$ & $110(31.1)$ & $97(27.4)$ & \\
\hline $\begin{array}{l}\text { ECOG Performance } \\
\text { Status }\end{array}$ & & & & 0.0044 \\
\hline $0-1$ & $317(51.4)$ & $152(24.6)$ & $148(24.0)$ & \\
\hline$\geq 2$ & $24(49.0)$ & $21(42.9)$ & $4(8.2)$ & \\
\hline AJCC Stage & & & & 0.0927 \\
\hline IIIB & $95(42.2)$ & $73(32.4)$ & $57(25.3)$ & \\
\hline IV & $314(50.3)$ & $184(29.5)$ & $126(20.2)$ & \\
\hline Histology subtype & & & & 0.0635 \\
\hline $\begin{array}{l}\text { Squamous cell } \\
\text { carcinoma }\end{array}$ & $85(41.1)$ & $71(34.3)$ & $51(24.6)$ & \\
\hline Non-Squamous & $331(50.4)$ & $193(29.4)$ & $133(20.2)$ & \\
\hline Site & & & & 0.1185 \\
\hline Primary tumor & $273(46.7)$ & $190(32.5)$ & $121(20.7)$ & \\
\hline Metastatic lesion & $151(51.2)$ & $76(25.8)$ & $68(23.1)$ & \\
\hline Tissue type & & & & 0.1899 \\
\hline Surgical resection & $161(51.3)$ & $82(26.1)$ & $71(22.6)$ & \\
\hline *Biopsy & $241(47.2)$ & $164(32.1)$ & $106(20.7)$ & \\
\hline Sampling location & & & & 0.3803 \\
\hline Lymph node & $67(50.0)$ & $32(23.9)$ & $35(26.1)$ & \\
\hline Brain & $32(55.2)$ & $14(24.1)$ & $12(20.7)$ & \\
\hline Liver & $6(66.7)$ & 0 & $3(33.3)$ & \\
\hline \#Others & 46 (48.9) & 30 (31.9) & 18 (19.2) & \\
\hline $\begin{array}{l}\text { TPS: tumor proport } \\
\text { * including biopsy s } \\
\text { biopsy, or EBUS/TE } \\
\text { \# Others indicate sa } \\
\text { tract, heart, kidney, } \\
\text { and other organs. }\end{array}$ & $\begin{array}{l}\text { iple from } \\
\text { A. } \\
\text { ples from }\end{array}$ & $\begin{array}{l}\text { astern Co } \\
\text { eedle biop } \\
\text { renal glan } \\
\text { pancreas, }\end{array}$ & $\begin{array}{l}\text { ve Oncolc } \\
\text { nchial Bic } \\
\text { ladder, g } \\
\text { thyroid, }\end{array}$ & $\begin{array}{l}\text { Jroup. } \\
\text { pleura } \\
\text { intestinal } \\
\text { a, soft tissue, }\end{array}$ \\
\hline
\end{tabular}

\section{Prevalence of PD-L1 in EGFR/ALK mutated NSCLC}

No significant difference in PD-L1 expression prevalence was observed for patients with different ALK mutation states, wild-type, or ALK translocations (Table 3).

Table 3. Prevalence of PD-L1 in EGFR/ALK-mutated NSCLC

\begin{tabular}{|c|c|c|c|c|}
\hline Category, N (\%) & TPS $<1 \%$ & TPS $1-49 \%$ & TPS $\geq 50 \%$ & P-value \\
\hline ALK status & & & & 0.9947 \\
\hline Rearrangement & $17(53.1)$ & $8(25.0)$ & 7 (21.9) & \\
\hline Wild type & $261(52.2)$ & $128(25.6)$ & $111(22.2)$ & \\
\hline EGFR mutation status & & & & 0.0017 \\
\hline Mutant & $118(56.2)$ & $62(29.5)$ & $30(14.3)$ & \\
\hline Wild type & $139(50.2)$ & $62(22.4)$ & $76(27.4)$ & \\
\hline Joint ALK/EGFR Status & & & & 0.0009 \\
\hline $\mathrm{ALK}^{+}$or $\mathrm{EGFR}^{+}$ & $108(56.8)$ & $57(30.0)$ & $25(13.2)$ & \\
\hline ALK- and EGFR- & $112(49.8)$ & $50(22.2)$ & $63(28.0)$ & \\
\hline
\end{tabular}

A slight difference was observed in EGFR mutant and wild-type groups when PD-L1 TPS $\geq 1 \%$ $(43.8 \%$ versus $49.8 \%)$. However, EGFR mutation status seemed to affect the proportion of PD-L1 expression $(p=0.0017)$. Patients with wild-type EGFR were more likely to have high PD-L1 expression than those with EGFR mutation (TPS $\geq 50 \%$ : $27.4 \%$ vs. 14.3\%). Likewise, patients with wild-type ALK and EGFR status had a higher likelihood of showing PD-L1 expression than those with EGFR or ALK alterations (TPS $\geq 1 \%$ : $50.2 \%$ vs. $43.2 \%$, TPS $\geq 50 \%$ : $28.0 \%$ vs. $13.2 \%$ ) (Table 3 ).

In addition, the distribution of EGFR/ALK status in different PD-L1 expression categories showed that samples with wild-type EGFR or ALK mutation occupied a higher percentage than mutated samples in all PD-L1 expression categories (Table 4).

Table 4. Distribution of EGFR/ALK status in different PD-LI expression categories

\begin{tabular}{llllllll}
\hline \begin{tabular}{l} 
Category, Total $\begin{array}{l}\text { EGFR } \\
\text { N }(\%)\end{array}$ \\
\cline { 3 - 7 }
\end{tabular} & & mutant & wild type & no data & rearrangement & $\begin{array}{l}\text { wild } \\
\text { type }\end{array}$ & no data \\
\hline TPS $<1 \%$ & 424 & 118 & $139(32.8)$ & 167 & $17(4.0)$ & 261 & 146 \\
& & $(27.8)$ & & $(39.4)$ & & $(61.6)$ & $(34.4)$ \\
TPS $\geq 1 \%$ & 455 & $92(20.2)$ & $138(30.3)$ & 225 & $15(3.3)$ & 239 & 201 \\
& & & & $(49.5)$ & & $(52.5)$ & $(44.2)$ \\
TPS $\geq$ & 189 & $30(15.9)$ & $76(40.2)$ & $83(43.9)$ & $7(3.7)$ & 111 & $71(37.6)$ \\
$50 \%$ & & & & & & $(58.7)$ & \\
\hline
\end{tabular}

\section{PD-L1 expression using $22 \mathrm{C} 3$ antibody concentrate}

Among 879 samples, $248 \quad(28.2 \%)$ were eventually tested using the 22C3 antibody concentrate. Of the 248 samples evaluated, 99 (40.0\%) were tested on the Dako ASL48 platform and 149 $(60.1 \%)$ were tested on the Ventana BenchMark platform. When compared to the overall staining results obtained with the PD-L1 IHC 22C3 pharmDx ${ }^{\mathrm{TM}}$ kit, LDT staining on the Dako ASL48 platform showed $100 \%$ concordance for TPS $<1 \%$, a slightly lower rate for TPS of $1-49 \%$ (23.2\% for LDT and $24.2 \%$ for the overall staining method), and a slightly higher rate for TPS $\geq 50 \%$ ( $29.3 \%$ for LDT and $28.3 \%$ for the overall staining method). On the contrary, LDT staining on the Ventana BenchMark platform showed a higher rate for TPS < $1 \%$ (40.9\% for LDT and $39.6 \%$ for the overall staining method) and TPS from $1-49 \%$ (34.9\% for LDT and $30.9 \%$ for the overall staining method) and a lower rate for TPS $\geq$ $50 \%$ (24.2\% for LDT and $29.5 \%$ for the overall staining method).

\section{Discussion}

The current real-world retrospective study explored the prevalence and features of PD-L1 tumor expression in a large-scale Chinese population with advanced NSCLC. We found that roughly half of the patients with advanced NSCLC (51.8\%) had a PD-L1 
expression TPS $\geq 1 \%$ and nearly one-fifth of patients $(21.5 \%)$ had high PD-L1 expression (TPS $\geq 50 \%$ ). PD-L1 expression was more likely to be found in individuals less than 75 years old, men, current/former smokers, those with good PS scores, and those with wild-type EGFR. PD-L1 expression determined using the $22 \mathrm{C} 3$ antibody concentrate and kit had comparable results.

The prevalence of positive PD-L1 expression in our Chinese cohort is largely consistent with that displayed in a global study. The EXPRESS study including tumor samples with a broad range of demographic and clinicopathological characteristics reported an overall $52 \%$ of PD-L1 TPS $\geq 1 \%$ and $22 \%$ of PD-L1 TPS $\geq 50 \%$ in patients with advanced NSCLC.[12] The corresponding values in the current study were $51.8 \%$ of PD-L1 TPS $\geq 1 \%$ and $21.5 \%$ of PD-L1 TPS $\geq 50 \%$.

Increasing evidence has demonstrated that PD-L1 expression related to aggressive behavior and disease progression may generally serve as a poor prognostic biomarker for patients with NSCLC [13]. First, PD-L1 expression was observed more frequently in the advanced stage of NSCLC [14-16]. For the NSCLC population in China, a previous small-scale study with surgically staged I-III samples showed that PD-L1 expression assessed using the PD-L1 IHC 22C3 antibody varied from $4.1 \%$ in adenocarcinomas to $34.3 \%$ in squamous cell carcinomas [17]. In another study including 305 Chinese patients with stage I-IV NSCLC, the prevalence of PD-L1 expression assessed with the $22 \mathrm{C} 3$ assay was $46.6 \%$ with $\geq 1 \%$ as the cutoff and $20.7 \%$ with $\geq 50 \%$ as the cutoff [18]. By contrast, the prevalence of PD-L1 expression, $\geq 1 \%$ or $\geq 50 \%$, seems to be higher in the current study, partly due to the fact that all measured samples were from the advanced III/IV stage. Second, PD-L1 expression was more likely to present in the aggressive solid subtype of adenocarcinomas, which was correlated with worse survival $[19,20]$. It was also associated with poor tumor differentiation and positive lymph nodal metastasis [13]. Third, in locally advanced stage NSCLC, PD-L1 expression predicted postoperative recurrence [21]. Fourth, in vivo and in vitro experiments confirmed that PD-L1 functioned as a tumor-promoting factor in lung cancer [22]. As single or combined immunotherapy has been approved for the first-line treatment of advanced NSCLC, our findings represent the real percentage of candidates who might benefit from indications for immunotherapy $[4,6,7,23,24]$.

There are controversies regarding the relationship between PD-L1 expression and demographic and clinicopathological features, which may be attributed to the population heterogeneity in various studies. Some earlier studies did not support an association between PD-L1 expression and gender, age, smoking history, or PS score [25]. We found that positive PD-L1 expression was more frequently observed in men, current/former smokers, and individuals with good PS scores. These findings were inconsistent with some previous reports [18, 26]. Tobacco exposure induces chronic inflammation and upregulates interferon- $\gamma$, which triggers increased PD-L1 expression [27, 28]. More specifically, higher daily cigarette consumption, longer smoking duration, and current smoking status were significantly associated with high PD-L1 expression [18]. Considering that men tend to smoke at higher rates than women, it is not surprising that men had a greater percentage of positive PD-L1 expression, as confirmed in this study [26, 29].

EGFR- or ALK-tyrosine kinase inhibitor (TKI) treatment is an important targeted therapy for lung adenocarcinoma in East Asian patients because the overall EGFR mutation frequency is $47.9 \%$ in Asian patients and only $19.2 \%$ in Western patients $[30,31]$. However, there is insufficient evidence to support the application of mono-immunotherapy for NSCLC patients who fail to respond to frontline TKI therapy [32-34]. The correlations between the EGFR/ALK mutation and PD-L1 expression may provide some clues for immunotherapy in this subgroup. Our findings validated previous findings that NSCLC patients with EGFR mutation had a lower chance of PD-L1 expression than those with wild-type EGFR [26, 33-36]. In the current study, 62 (29.5\%) NSCLC patients with EGFR mutations had a PD-L1 expression of $1 \%-49 \%$ and $30(14.3 \%)$ had PD-L1 expression $\geq 50 \%$. The proportion of positive PD-L1 expression was similar to previous findings [37]. The overlap between PD-L1 expression and EGFR mutations is still considerable based on the results from a previous study in Chinese lung adenocarcinoma patients [19]. The subgroup of lung adenocarcinomas patients with co-occurrence of aberrations in classical therapeutic genes such as EGFR mutation and PD-L1 positive expression accounted for $9.6 \%$ of Chinese adenocarcinoma patients [19]. Therefore, whether immunotherapy combined with chemotherapy or anti-angiogenesis agent could be an effective option for EGFR-mutated NSCLC after exhaustion of targeted therapies is under research in ongoing KEYNOTE-789 (NCT03515837) and Checkmate-722 (NCT02864251) and ORIENT-31 (NCT03802240) studies. Another issue worth clarifying is that the EGFR/ALK mutation status was retrospectively collected in this study. Among the available detection methods, NGS may have advantages over PCR by providing a higher mutation 
detection rate and a wider spectrum. However, given that more than $90 \%$ of detected EGFR mutations were common mutations of $19 \mathrm{del}$ or L858R that could be well identified by all methods; we speculate that our findings are applicable in different settings [38].

Although a lower PD-L1 positive rate was associated with EGFR mutation, NSCLC patients with ALK rearrangement were reported to possess higher PD-L1 expression $[36,39]$. Research findings showing that the EML4-ALK oncoprotein can upregulate PD-L1 expressions in lung cancer cells explain this result $[40,41]$. Recent studies showed that 50\%-55.6\% of ALK-positive NSCLC patients were PD-L1-positive (TPS $\geq 1 \%$ ) and $16-30.6 \%$ had high expression (TPS $\geq$ $50 \%)[39,42]$. We also observed a high percentage of TPS $\geq 50 \% \quad(21.9 \%)$ in ALK-rearranged NSCLC. However, no significant difference was seen between patients with wild-type or rearranged ALK status. In addition, clinical trials demonstrated that the combination of ALK-TKI and immunotherapy may lead to higher toxicity [43]. Whether a higher rate of positive PD-L1 expression implies a higher possibility of immunotherapy remains unclear for ALK-positive NSCLC patients.

The occurrence of brain metastasis in NSCLC is steadily increasing with advances in therapeutic strategies [44, 45]. The prognosis of patients with brain metastases remains poor [46]. Patients with brain metastases are mostly excluded from pivotal clinical trials and the prevalence of PD-L1 expression in metastatic brain tissue is largely unknown. In this study, we obtained 58 brain metastatic tissue samples, $44.8 \%$ of which exhibited PD-L1 expression (TPS $\geq$ $1 \%$ ) while $20.7 \%$ exhibited high expression (TPS $\geq 50 \%$ ). The expression pattern of PD-L1 did not differ from those of other sample tissues. A recent study reported concordant PD-L1 expression between brain metastases and primary tumors [47]. However, the prognostic role of PD-L1 expression in brain metastases has not been fully discovered although encouraging results suggest that immunotherapy may be active in the central nervous system (CNS) in NSCLC patients with high PD-L1 expression [48, 49].

PD-L1 IHC 22C3 pharmDx is a companion diagnostic test for PD-L1 detection. Different PD-L1 assays and evaluation systems for PD-L1 expression may produce discrepant results [50, 51]. Other IHC assays using antibodies instead of the $22 \mathrm{C} 3$ clone, for example, 28-8, SP263, and SP142, have been developed to evaluate PD-L1 expression and are commercially available. High concordance for PD-L1 staining can be achieved between PD-L1 IHC 22C3 pharmDx assay and LDTs [52]. Additionally, both Ventana stains (UltraView and OptiView) have a high correlation with the PD-L1 IHC 22C3 pharmDx kit
[53]. Part of the tumor samples in this study were tested using 22C3 antibody concentrate. The findings that PD-L1 expression determined using the 22C3 antibody concentrate on the Dako ASL48 platform and Ventana BenchMark Platform was comparable to that determined with validated LDTs support the feasibility of the clinical application of these methods.

There are several limitations in this study. First, the retrospective nature limited the comprehensiveness of the data. Second, we did not further evaluate the association between different subtypes of EGFR/ALK mutations and PD-L1, and other important driver genes such as KRAS were not analyzed. Third, the concordance of the 22C3 antibody concentrate and traditional assay kit on different platforms is worthy of further validation in larger sample sets.

In conclusion, the prevalence of PD-L1 expression in Chinese patients with advanced NSCLC is consistent with that reported in the global EXPRESS study. Age, gender, smoking history, PS scores, and EGFR/ALK mutation status affected PD-L1 expression. The $22 \mathrm{C} 3$ antibody concentrate may be an alternative regent for the $22 \mathrm{C} 3$ pharmDx assay.

\section{Abbreviations}

NSCLC: non-small cell lung cancer; ICI: immune checkpoint inhibitor; FDA: Food and Drug Administration; PD-1: programmed-cell death-1; PD-L1: programmed-cell death-1 ligand; IHC: immunohistochemical; LDTs: validated laboratory-developed tests; EGFR: epidermal growth factor receptor; ALK: anaplastic lymphoma kinase; FFPE: formalin-fixed paraffin-embedded; TPS: tumor proportion score; ECOG PS: Eastern Cooperative Oncology Group Performance Status; CNS: central nervous system.

\section{Author contribution}

Yan Jin, Jingping Yun, Chunyan Wu, Wenyong Sun, Anhua Mao, Wenmin Tang, Suijun Xiao, Yuan Li and Dongmei Lin conceived, designed, or planned the study. Xin Yang, Lili Jiang, Yan Jin, Peng Li, Yingyong Hou, Jingping Yun, Chunyan $\mathrm{Wu}$, Wenyong Sun, Xiangshan Fan, Dong Kuang, Weiya Wang, Jinsong $\mathrm{Ni}$ and Yuan Li collected and assembled the data. Yan Jin, Jingping Yun, Weiya Wang, Zhenhua Liu, Jiali Wang, Suijun Xiao, Jinsong Ni, Yuan Li and Dongmei Lin performed or supervised the analyses. Lili Jiang, Jingping Yun, Xiangshan Fan, Weiya Wang, Jinsong $\mathrm{Ni}$, Zhenhua Liu, Jiali Wang, Suijun Xiao, Yuan Li and Dongmei Lin interpreted the results. Yan Jin, Jingping Yun and Yuan Li wrote sections of the initial draft. Anhua Mao and Yuan Li provided administrative, technical, and logistic support. All authors provided 
substantive suggestions for revisions, reviewed and approved the final version of the paper, and ensured the accuracy of all aspects of the work.

\section{Acknowledgments}

Editorial assistance was provided by Medjaden Inc. This assistance was funded by MSD China.

\section{Funding statement}

The study was funded by MSD China.

\section{Competing Interests}

Anhua Mao, Wenmin Tang, Zhenhua Liu, Jiali Wang and Suijun Xiao are employees of MSD China. Other authors declare that they have no competing interests.

\section{References}

1. Siegel RL, Miller KD, Jemal A. Cancer statistics, 2020. CA Cancer J Clin. 2020; 70: 7-30

2. Chen W, Zheng R, Baade PD, et al. Cancer statistics in China, 2015. CA Cancer J Clin. 2016; 66: 115-32

3. Fukuoka M, Wu YL, Thongprasert S, et al. Biomarker analyses and final overall survival results from a phase III, randomized, open-label, first-line study of gefitinib versus carboplatin/paclitaxel in clinically selected patients with advanced non-small-cell lung cancer in Asia (IPASS). J Clin Oncol. 2011; 29: 2866-74.

4. Mok TSK, Wu YL, Kudaba I, et al. Pembrolizumab versus chemotherapy for previously untreated, PD-L1-expressing, locally advanced or metastatic non-small-cell lung cancer (KEYNOTE-042): a randomised, open-label, controlled, phase 3 trial. Lancet. 2019; 393: 1819-30.

5. Reck M, Rodríguez-Abreu D, Robinson AG, et al. Updated Analysis of KEYNOTE-024: Pembrolizumab Versus Platinum-Based Chemotherapy for Advanced Non-Small-Cell Lung Cancer With PD-L1 Tumor Proportion Score of $50 \%$ or Greater. J Clin Oncol. 2019; 37: 537-46.

6. Borghaei H, Langer CJ, Gadgeel S, et al. 24-Month Overall Survival from KEYNOTE-021 Cohort G: Pemetrexed and Carboplatin with or without Pembrolizumab as First-Line Therapy for Advanced Nonsquamous Non-Small Cell Lung Cancer. J Thorac Oncol. 2019; 14: 124-9.

7. Gandhi L, Rodríguez-Abreu D, Gadgeel S, et al. Pembrolizumab plus Chemotherapy in Metastatic Non-Small-Cell Lung Cancer. N Engl J Med. 2018; 378: 2078-92.

8. Paz-Ares L, Luft A, Vicente D, et al. Pembrolizumab plus Chemotherapy for Squamous Non-Small-Cell Lung Cancer. N Engl J Med. 2018; 379: 2040-51.

9. Sunshine J, Taube JM. PD-1/PD-L1 inhibitors. Curr Opin Pharmacol. 2015; 23: $32-8$

10. Garon EB, Rizvi NA, Hui R, et al. Pembrolizumab for the treatment of non-small-cell lung cancer. N Engl J Med. 2015; 372: 2018-28.

11. Deng HY, Zheng $X$, Zha $P$, et al. Diabetes mellitus and survival of non-small cell lung cancer patients after surgery: A comprehensive systematic review and meta-analysis. Thorac Cancer. 2019; 10: 571-8.

12. Dietel M, Savelov N, Salanova R, et al. Real-world prevalence of programmed death ligand 1 expression in locally advanced or metastatic non-small-cell lung cancer: The global, multicenter EXPRESS study. Lung Cancer. 2019; 134: 174-9.

13. $\mathrm{Li} \mathrm{H}, \mathrm{Xu} \mathrm{Y}$, Wan $\mathrm{B}$, et al. The clinicopathological and prognostic significance of PD-L1 expression assessed by immunohistochemistry in lung cancer: a meta-analysis of 50 studies with 11,383 patients. Transl Lung Cancer Res. 2019; 8: 429-49.

14. Wang H, Agulnik J, Kasymjanova G, et al. Cytology cell blocks are suitable for immunohistochemical testing for PD-L1 in lung cancer. Ann Oncol. 2018; 29: 1417-22.

15. Skov BG, Rørvig SB, Jensen THL, et al. The prevalence of programmed death ligand-1 (PD-L1) expression in non-small cell lung cancer in an unselected, consecutive population. Mod Pathol. 2020; 33: 109-17.

16. Chen $\mathrm{YB}, \mathrm{Mu} \mathrm{CY}$, Huang JA. Clinical significance of programmed death-1 ligand-1 expression in patients with non-small cell lung cancer: a 5-year-follow-up study. Tumori. 2012; 98: 751-5.

17. Pan $\mathrm{Y}$, Zheng $\mathrm{D}, \mathrm{Li} \mathrm{Y}$, et al. Unique distribution of programmed death ligand 1 (PD-L1) expression in East Asian non-small cell lung cancer. J Thorac Dis. 2017; 9: 2579-86

18. Song P, Guo L, Li W, et al. Clinicopathologic Correlation With Expression of PD-L1 on Both Tumor Cells and Tumor-infiltrating Immune Cells in Patients With Non-Small Cell Lung Cancer. J Immunother. 2019; 42: 23-8.
19. Wu J, Sun W, Wang $\mathrm{H}$, et al. The correlation and overlaps between PD-L1 expression and classical genomic aberrations in Chinese lung adenocarcinoma patients: a single center case series. Cancer Biol Med. 2019; 16: 811-21.

20. Da Cruz V, Yvorel V, Casteillo F, et al. Histopathological subtyping is a prognostic factor in stage IV lung adenocarcinoma. Lung Cancer. 2020; 147: 77-82.

21. Teramoto $\mathrm{K}$, Igarashi $\mathrm{T}$, Kataoka $\mathrm{Y}$, et al. Biphasic prognostic significance of PD-L1 expression status in patients with early- and locally advanced-stage non-small cell lung cancer. Cancer Immunol Immunother. 2021; 70: 1063-74.

22. Yu W, Hua Y, Qiu H, et al. PD-L1 promotes tumor growth and progression by activating WIP and $\beta$-catenin signaling pathways and predicts poor prognosis in lung cancer. Cell Death Dis. 2020; 11: 506.

23. Reck M, Rodríguez-Abreu D, Robinson AG, et al. Pembrolizumab versus Chemotherapy for PD-L1-Positive Non-Small-Cell Lung Cancer. N Engl J Med. 2016; 375: 1823-33.

24. Gadgeel S, Rodríguez-Abreu D, Speranza G, et al. Updated Analysis From KEYNOTE-189: Pembrolizumab or Placebo Plus Pemetrexed and Platinum for Previously Untreated Metastatic Nonsquamous Non-Small-Cell Lung Cancer. J Clin Oncol. 2020; 38: 1505-17.

25. Brody R, Zhang Y, Ballas M, et al. PD-L1 expression in advanced NSCLC: Insights into risk stratification and treatment selection from a systematic literature review. Lung Cancer. 2017; 112: 200-15.

26. Tseng JS, Yang TY, Wu CY, et al. Characteristics and Predictive Value of PD-L1 Status in Real-World Non-Small Cell Lung Cancer Patients. J Immunother. 2018; 41: 292-9.

27. Conway EM, Pikor LA, Kung SH, et al. Macrophages, Inflammation, and Lung Cancer. Am J Respir Crit Care Med. 2016; 193: 116-30.

28. Sun C, Mezzadra R, Schumacher TN. Regulation and Function of the PD-L1 Checkpoint. Immunity. 2018; 48: 434-52.

29. Higgins ST, Kurti AN, Redner R, et al. A literature review on prevalence of gender differences and intersections with other vulnerabilities to tobacco use in the United States, 2004-2014. Prev Med. 2015; 80: 89-100.

30. Dearden S, Stevens J, Wu YL, et al. Mutation incidence and coincidence in non small-cell lung cancer: meta-analyses by ethnicity and histology (mutMap). Ann Oncol. 2013; 24: 2371-6.

31. Zhou F, Zhou C. Lung cancer in never smokers-the East Asian experience. Transl Lung Cancer Res. 2018; 7: 450-63.

32. Socinski MA, Jotte RM, Cappuzzo F, et al. Atezolizumab for First-Line Treatment of Metastatic Nonsquamous NSCLC. N Engl J Med. 2018; 378: 2288-301.

33. Hsu KH, Huang YH, Tseng JS, et al. High PD-L1 expression correlates with primary resistance to EGFR-TKIs in treatment naïve advanced EGFR-mutant lung adenocarcinoma patients. Lung Cancer. 2019; 127: 37-43.

34. Yang CY, Liao WY, Ho CC, et al. Association between programmed death-ligand 1 expression, immune microenvironments, and clinical outcomes in epidermal growth factor receptor mutant lung adenocarcinoma patients treated with tyrosine kinase inhibitors. Eur J Cancer. 2020; 124: 110-22.

35. Jin Y, Shen X, Pan Y, et al. Correlation between PD-L1 expression and clinicopathological characteristics of non-small cell lung cancer: A real-world study of a large Chinese cohort. J Thorac Dis. 2019; 11: 4591-601.

36. Lee J, Park CK, Yoon HK, et al. PD-L1 expression in ROS1-rearranged non-small cell lung cancer: A study using simultaneous genotypic screening of EGFR, ALK, and ROS1. Thorac Cancer. 2019; 10: 103-10.

37. Yoneshima $\mathrm{Y}$, Ijichi K, Anai S, et al. PD-L1 expression in lung adenocarcinoma harboring EGFR mutations or ALK rearrangements. Lung Cancer. 2018; 118: $36-40$

38. Mao L, Zhao W, Li X, et al. Mutation Spectrum of EGFR From 21,324 Chinese Patients With Non-Small Cell Lung Cancer (NSCLC) Successfully Tested by Multiple Methods in a CAP-Accredited Laboratory. Pathol Oncol Res. 2021; 27: 602726 .

39. Evans M, O'Sullivan B, Hughes F, et al. The Clinicopathological and Molecular Associations of PD-L1 Expression in Non-small Cell Lung Cancer: Analysis of a Series of 10,005 Cases Tested with the 22C3 Assay. Pathol Oncol Res. 2020; 26: 79-89.

40. Koh J, Jang JY, Keam B, et al. EML4-ALK enhances programmed cell death-ligand 1 expression in pulmonary adenocarcinoma via hypoxia-inducible factor (HIF)-1a and STAT3. Oncoimmunology. 2016; 5: e1108514.

41. Ota K, Azuma K, Kawahara A, et al. Induction of PD-L1 Expression by the EML4-ALK Oncoprotein and Downstream Signaling Pathways in Non-Small Cell Lung Cancer. Clin Cancer Res. 2015; 21: 4014-21.

42. Chang GC, Yang TY, Chen KC, et al. ALK variants, PD-L1 expression, and their association with outcomes in ALK-positive NSCLC patients. Sci Rep. 2020; 10: 21063.

43. Felip E, de Braud FG, Maur M, et al. Ceritinib plus Nivolumab in Patients with Advanced ALK-Rearranged Non-Small Cell Lung Cancer: Results of an Open-Label, Multicenter, Phase 1B Study. J Thorac Oncol. 2020; 15: 392-403.

44. Ostrom QT, Wright $\mathrm{CH}$, Barnholtz-Sloan JS. Brain metastases: epidemiology. Handb Clin Neurol. 2018; 149: 27-42.

45. Cagney DN, Martin AM, Catalano PJ, et al. Incidence and prognosis of patients with brain metastases at diagnosis of systemic malignancy: a population-based study. Neuro Oncol. 2017; 19: 1511-21.

46. Achrol AS, Rennert RC, Anders $C$, et al. Brain metastases. Nat Rev Dis Primers. 2019; 5: 5 
47. Camy F, Karpathiou G, Dumollard JM, et al. Brain metastasis PD-L1 and CD8 expression is dependent on primary tumor type and its PD-L1 and CD8 status. J Immunother Cancer. 2020; 8: e000597.

48. Eguren-Santamaria I, Sanmamed MF, Goldberg SB, et al. PD-1/PD-L1 Blockers in NSCLC Brain Metastases: Challenging Paradigms and Clinical Practice. Clin Cancer Res. 2020; 26: 4186-97.

49. Goldberg SB, Gettinger SN, Mahajan A, et al. Pembrolizumab for patients with melanoma or non-small-cell lung cancer and untreated brain metastases: early analysis of a non-randomised, open-label, phase 2 trial. Lancet Oncol. 2016; 17: 976-83.

50. Krigsfeld GS, Prince EA, Pratt J, et al. Analysis of real-world PD-L1 IHC 28-8 and $22 \mathrm{C} 3$ pharmDx assay utilisation, turnaround times and analytical concordance across multiple tumour types. J Clin Pathol. 2020; 73: 656-64.

51. Jiang $\mathrm{L}, \mathrm{Su} X, \mathrm{Zhang} \mathrm{T}$, et al. PD-L1 expression and its relationship with oncogenic drivers in non-small cell lung cancer (NSCLC). Oncotarget. 2017; 8: 26845-57.

52. Ilie M, Khambata-Ford S, Copie-Bergman C, et al. Use of the $22 \mathrm{C} 3$ anti-PD-L1 antibody to determine PD-L1 expression in multiple automated immunohistochemistry platforms. PLoS One. 2017; 12: e0183023.

53. Neuman T, London M, Kania-Almog J, et al. A Harmonization Study for the Use of 22C3 PD-L1 Immunohistochemical Staining on Ventana's Platform. J Thorac Oncol. 2016; 11: 1863-8. 